\title{
Crisis humanitaria de emergencia en Colombia por violencia contra las mujeres durante la pandemia de COVID-19
}

\author{
The emergency humanitarian crisis in Colombia due to \\ violence against women during the COVID-19 pandemic
}

\section{Gladys Rocío Ariza-Sosa}

Doctora en Salud Pública

Universidad de Antioquia - Colombia

Líder del grupo de investigación Respuesta Social en Salud

Correo electrónico: gladys.ariza@udea.edu.co

\begin{abstract}
Juan Jacobo Agudelo-Galeano
Magíster en Estudios Políticos

Fundación Universitaria Claretiana - Colombia

Líder del grupo de investigación Humanidades y Estudios Socioculturales

Correo electrónico: investigacionhcr@uniclaretiana.edu.co
\end{abstract}

\section{Luisa Alejandra Saldarriaga-Quintero}

Magíster en Educación

Universidad Católica de Oriente - Colombia

Investigadora asociada, Grupo de Investigaciones Jurídicas

Correo electrónico: Isaldarriaga@uco.edu.co 


\title{
María Camila Ortega-Mosquera
}

\author{
Especialista en Derecho Médico - Colombia \\ Asesora jurídica de unidad de apoyo, Concejo de Medellín \\ Correo electrónico: camila-2197@outlook.com
}

\section{Dora Cecilia Saldarriaga-Grisales}

\author{
Magíster en Derechos Humanos y Democratización \\ Universidad Autónoma Latinoamericana, UNAULA - Colombia \\ Investigadora asociada, Grupo de Investigación en Constitucionalismo Crítico y Género \\ Correo electrónico doraceciliasaldarriaga@gmail.com
}

\section{Resumen}

La violencia contra las mujeres en 2020 ha aumentado durante el confinamiento por la pandemia de COVID-19. Se presenta un artículo de revisión narrativa, con el objetivo de analizar por qué es procedente declarar la crisis humanitaria de emergencia en Colombia por violencia contra las mujeres durante la pandemia. La revisión se realizó a partir de búsquedas sistemáticas en bases de datos. La violencia contra las mujeres, en esta contingencia, ha tenido consecuencias desde lo social y familiar, hasta lo psicológico, estas últimas más visibles con el aumento de la ansiedad, la depresión, la sensación de culpabilidad y los suicidios. En el contexto de la pandemia, el Gobierno colombiano ha expedido normas que pretenden reforzar obligaciones que se encuentran incorporadas en el ordenamiento jurídico nacional. Sin embargo, las barreras estructurales y las brechas de género continúan obstaculizando el libre acceso de las mujeres colombianas a la justicia, lo que impide la plena garantía de sus derechos humanos. Las violencias contra las mujeres en Colombia, en 2020, se configuran como una crisis humanitaria por su inadecuada atención y por el creciente número de casos.

\section{Cómo citar este artículo:}

Ariza-Sosa, G., Agudelo-Galeano, J., Saldarriaga-Quintero, L., Ortega-Mosquera, M., \& SaldarriagaGrisales, D. (2021). Crisis humanitaria de emergencia en Colombia por violencia contra las mujeres durante la pandemia de COVID-19. Revista de la Facultad de Derecho y Ciencias Políticas, 51 (134), pp. $125-150$.

doi: https://doi.org/10.18566/rfdcp.v51n134.a06

Recibido: 12 de julio de 2020

Aprobado: 19 de noviembre de 2020 


\section{Palabras clave}

Violencia contra la mujer; infecciones por coronavirus; pandemias Colombia (descriptores DeCS); Crisis política (descriptor UNESCO).

\section{Abstract}

Violence against women in 2020 has increased during lockdown from the COVID-19 pandemic. We present a narrative review article to analyze why it is appropriate to declare the humanitarian crisis of emergency in Colombia due to violence against women during the pandemic. We reviewed systematic searches in databases. Violence against women, in this contingency, has had consequences from the social and family, to the psychological, the latter more visible with the increase in anxiety, depression, the feeling of guilt, and suicides. In the pandemic context, the Colombian government has issued regulations that seek to reinforce obligations incorporated into the national legal system. However, structural barriers and gender gaps continue to hinder Colombian women's free access to justice. It prevents the full guarantee of their human rights. Violence against women in Colombia, in 2020, configures as a humanitarian crisis due to its inadequate attention and the growing number of cases.

\section{Keywords}

Violence against women; coronavirus infections; Colombia pandemics (DCS descriptors); Political crisis (UNESCO descriptor).

\section{Introducción}

La emergencia originada por la COVID-19 se presenta en un momento en el que la humanidad parecía sentirse invencible. Se ha constituido como una emergencia sanitaria global sin precedentes en el último siglo, aunque mediada por el acceso a las tecnologías, las cuales dan un matiz diferente a las experiencias humanas contemporáneas.

Si bien la violencia contra las mujeres y las niñas no es un problema social nuevo, durante la pandemia de COVID 19, se han incrementado las estadísticas respecto a este fenómeno de manera alarmante en todo el mundo. Incluso se afirma que se han presentado retrasos en victorias logradas durante décadas por los movimientos de mujeres, como lo señaló António Guterres (UN, 2020) en su discurso ante la Asamblea General de la ONU, el 22 de septiembre de 2020: 
Media humanidad está soportando las peores consecuencias sociales y económicas de la COVID-19. Las mujeres están representadas desproporcionadamente en los sectores más afectados por la pérdida de empleos. Son también quienes realizan la mayor parte del trabajo de cuidado no remunerado generado por la pandemia. Y son, además, quienes tienen menos recursos económicos a los que recurrir, porque sus salarios son más bajos y tienen menos acceso a beneficios. Al mismo tiempo, millones de niñas están perdiendo la oportunidad de recibir una educación y tener un futuro, por el cierre de las escuelas y el alza del matrimonio infantil. A menos que actuemos ahora, la igualdad de género podría retroceder décadas. También debemos erradicar el espantoso aumento de la violencia contra las mujeres y las niñas durante la pandemia, desde la violencia doméstica hasta el abuso sexual, el acoso en línea y el femicidio. Esta es una guerra oculta contra las mujeres (versión en español, párr. 80).

En esta emergencia sanitaria en 116 de 178 países de los cuales se tienen datos, se ha implementado el confinamiento en los hogares como estrategia de salud pública para reducir la tasa de contagio, durante periodos de tiempo variables en 2020 (Hale et al., 2020). Esto ha expuesto a mujeres y a niñas a mayores cargas de trabajo doméstico en sus hogares, a pasar mucho más tiempo con los hombres de la familia, en medio de mayores tensiones por el encierro en espacios, muchas veces en condiciones de hacinamiento, con las privaciones materiales y de alimentos, resultado de la pérdida masiva de empleos y otras fuentes de ingresos. En consecuencia, se han reportado incrementos en las diferentes formas de violencia contra las mujeres: física, sexual, económica y psicológica, en diversos países como España (Gallo Rivera \& Mañas Alcón, 2020), Argentina (Carrasco \& Martínez Reina, 2020, p. 48-50), en Ecuador (Chamba-Parra et al., 2020), México (Casique, 2020), así como en otros países de América Latina y el Caribe (Castro, 2020, p. 18-25). Esto se ha evidenciado en un aumento de llamadas a líneas de atención a mujeres y de uso de casas de refugio, incremento de denuncias por violencia sexual, así como mayores cifras de feminicidios y desapariciones de mujeres (Llerena \& Narváez, 2020).

Las explicaciones dadas para la violencia en las relaciones de pareja antes de la pandemia siguen teniendo vigencia. En el contexto cultural, la inequidad de género es la condición estructural; en el contexto político, persiste la dominación masculina; y en el económico, la división sexual del trabajo (Ariza, 2013). Otra condición estructural se ubica en la misoginia promovida por el patriarcado como parte del contrato social (Trujillo, 2019). En este confinamiento domiciliario, como condiciones coyunturales que atizan el 
fenómeno de la violencia contra las mujeres, se encuentra que ellas, además de tener más trabajo porque ahora los integrantes de la familia pasan más tiempo en casa, deben asistir a sus hijos en la formación escolar a distancia sin dejar de lado las tareas del cuidado, limpieza y preparación de alimentos. También se ven obligadas a generar ingresos para el sostenimiento familiar (Unicef, 2020).

Debido a lo anterior, el aumento de la violencia contra las mujeres y las niñas, documentado en gran parte de los territorios mundiales, permite aludir a este complejo problema como la otra pandemia, la cual, aunque sin ser causada por un agente infeccioso, se ha extendido con mayor rapidez que en años previos. Esta violencia genera notable preocupación en entidades internacionales, así como en las organizaciones gubernamentales y de la sociedad civil, dado que restringe los derechos humanos a la libertad, la dignidad e incluso la vida de las mujeres. Más aún, se puede afirmar, que la violencia patriarcal es una endemia con la que la humanidad ha lidiado hace varios siglos.

Entre los efectos cognitivos y emocionales sufridos por las mujeres producto de la violencia se encuentran la depresión, el estrés, la ansiedad generalizada, el consumo de alcohol y otras sustancias psicoactivas. Debido al confinamiento, el agresor puede controlar más a su víctima, limitando en mayor proporción sus movimientos y su libertad. Aunado a ello, la pérdida de ingresos y del trabajo hacen que el agresor pretenda desquitarse con su pareja de forma más continua y constante. Como consecuencia, las mujeres afectadas pueden sufrir desestabilización emocional y un debilitamiento de la autoestima que se traduce en trastornos de ansiedad, fobias, depresión, disfunciones sexuales y diversas alteraciones en los rasgos de personalidad (Ballester \& Villanueva, 2014).

Según Perela (2010), la violencia psicológica se desarrolla en tres esferas: en la esfera social, cuando la mujer rompe con las relaciones construidas; en la esfera familiar, de amistades y trabajo; y de ruptura con su identidad. La víctima suspende recuerdos y relaciones pasadas. También sufre su identidad presente, a través de las críticas, los señalamientos y la minimización de sus gustos, aficiones, deseos e iniciativas, cuestionamientos provenientes del agresor. Estas afectaciones psicológicas se han evidenciado más en los últimos años, quizás por un mayor interés previo en visibilizar la violencia física, aparentemente más nefasta y manifiesta. Incluso las víctimas, con frecuencia, no se percatan de que están siendo violentadas. 
En este sentido, nuestro artículo presenta los resultados de una revisión que tiene como objetivo analizar por qué es necesario declarar la crisis humanitaria de emergencia en Colombia por violencia contra las mujeres durante la pandemia de COVID-19. La denominación de crisis humanitaria se hace en términos políticos, porque conlleva la declaración del gobierno o de las organizaciones internacionales para visibilizar la afectación generalizada de derechos humanos. En sede constitucional se reconoce como estado de cosas inconstitucional, por tanto, el artículo analiza, primero, la crisis humanitaria conceptualmente, y luego, los elementos que ha determinado la Corte para el segundo. Ambos conceptos son similares, solo que su campo de enunciación es diferente.

En este orden de ideas, las características que marcan las diferencias entre los sexos se producen a través de las teorías y prácticas de la política, como lo señaló Joan Scott (2008). Tanto el sexo como el género son conceptos que se han construido histórica y discursivamente. Ambos son formas de conocimiento, de significado, organizaciones de la percepción, estrechamente imbricadas. En tanto construcción, el género es un fenómeno psicosocial, histórico, complejo y cambiante. Scott precisa: "El género, las normas sociales que intentan organizar las relaciones de los hombres y las mujeres en las sociedades, produce el conocimiento que tenemos sobre el sexo y sobre la diferencia sexual (en nuestra cultura equiparando al sexo con la naturaleza)” (p. 248).

Inicialmente se exponen los métodos. Luego, se presenta el tema de las afectaciones en la salud mental de las mujeres víctimas, debido que develan una similitud entre el confinamiento y los desastres naturales y los conflictos, lo cual es uno de los múltiples argumentos para sustentar la declaración de crisis humanitaria. En la tercera sección se despliega un examen de los decretos legislativos en Colombia durante la pandemia con respecto al acceso a la justicia para las mujeres afectadas. Se finaliza con el análisis de las razones que fundamentan la declaración de una crisis humanitaria de emergencia por violencia contra las mujeres en el contexto de la pandemia por COVID-19 en Colombia.

\section{Métodos}

Con el objetivo de analizar por qué es necesario declarar la crisis humanitaria de emergencia en Colombia por violencia contra las mujeres durante la pandemia de COVID-19, se efectuó una revisión narrativa (Lozano, 2005). 
En la primera parte, para describir los efectos psicológicos de las mujeres víctimas de violencia de género en tiempos de pandemia, producto del confinamiento decretado por los gobiernos de carácter internacional, se hizo una búsqueda sistemática en bases de datos con acceso libre como Redalyc, Scielo, Dialnet, REDIB y Google Académico, en idioma español, con los términos COVID-19, pandemia, violencia, mujeres y psicología, de documentos publicados a partir del mes de marzo de 2020. Se recuperaron 14 textos, de los cuales se seleccionaron, de acuerdo con el contenido, cinco (5) para su análisis.

En lo relativo al acceso a la justicia se hizo también una búsqueda sistemática en Google académico, Vlex y Notinet con los términos "violencia contra las mujeres", pandemia, "COVID-19", sin restricción temporal, con lo cual se recuperaron 22 resultados enmarcados en el 2020, los cuales se filtraron de acuerdo con el contenido, para finalmente incluir 12 documentos.

Por último, en lo que atiene a la crisis humanitaria, la búsqueda sistemática con los términos "crisis humanitaria," "derechos humanos" se realizó en Google académico, para el periodo 2010 - 2020, con lo cual se recuperaron 6210 documentos. Frente a este escenario, se procedió a excluir: i) aquellos que hacían referencia a territorios específicos; ii) los que hacían referencia a temáticas por fuera de los derechos humanos; iii) los que no tenían una fundamentación teórica de crisis humanitaria. Así, se obtuvieron diez documentos que abordaban, de manera histórica y conceptual, la crisis humanitaria, y tras su lectura completa se incluyeron tres documentos. De igual forma, se revisó la línea jurisprudencial sobre el estado de cosas inconstitucional y se peticionó a las autoridades competentes para tener la información estadística de las violencias contra las mujeres en Colombia.

\section{Dimensión psicológica de la violencia de género en la pandemia}

La información y tratamiento de la dimensión psicológica de la violencia de género durante la pandemia de COVID-19 es incipiente. Las publicaciones al respecto mencionan las alteraciones emocionales o conductuales en la población general o plantean datos en relación con el incremento de las denuncias y la inocua e indiferente respuesta institucional vinculada a las diferentes formas de violencia. Es importante señalar la diversidad tipológica de los cinco documentos seleccionados y su alcance exploratorio. Uno de los 
textos refiere a los efectos generales desde lo emocional y conductual en la población en general, otros dos enfatizan la respuesta institucional frente a la situación en términos de la violencia psicológica, y los dos últimos plantean el riesgo y los factores de vulnerabilidad frente a la violencia de género.

En la tabla 1 se resumen las principales características de las investigaciones localizadas.

Tabla 1. Artículos seleccionados sobre violencia psicológica contra las mujeres.

\begin{tabular}{|l|l|l|l|l|l|}
\hline \multicolumn{1}{|c|}{$\begin{array}{c}\text { Título del } \\
\text { documento }\end{array}$} & $\begin{array}{c}\text { Tipo de } \\
\text { texto }\end{array}$ & País & $\begin{array}{c}\text { Tipo de es- } \\
\text { tudio }\end{array}$ & $\begin{array}{l}\text { Técnica de } \\
\text { recolección }\end{array}$ & $\begin{array}{c}\text { Efectos } \\
\text { psicológicos }\end{array}$ \\
\hline $\begin{array}{l}\text { Las consecuencias } \\
\text { psicológicas de } \\
\text { la COVID-19 y el } \\
\text { confinamiento }\end{array}$ & $\begin{array}{l}\text { Informe de } \\
\text { investiga- } \\
\text { ción }\end{array}$ & España & Enfoque mixto & $\begin{array}{l}\text { Entrevista } \\
\text { (primera } \\
\text { fase). } \\
\text { Encuesta } \\
\text { (segunda } \\
\text { fase) }\end{array}$ & $\begin{array}{l}\text { Alteraciones } \\
\text { emocionales o } \\
\text { conductuales }\end{array}$ \\
\hline $\begin{array}{l}\text { ¿Recrudecimiento } \\
\text { de la violencia } \\
\text { hacia las mujeres } \\
\text { en los hogares } \\
\text { durante la } \\
\text { cuarentena por el } \\
\text { COVID-19? }\end{array}$ & $\begin{array}{l}\text { Nota } \\
\text { reflexiva }\end{array}$ & México & $\begin{array}{l}\text { Investigación } \\
\text { documental }\end{array}$ & $\begin{array}{l}\text { Revisión } \\
\text { documental }\end{array}$ & $\begin{array}{l}\text { Poca solicitud de } \\
\text { ayuda por parte } \\
\text { de las mujeres } \\
\text { por inadecuada } \\
\text { respuesta } \\
\text { institucional }\end{array}$ \\
\hline $\begin{array}{l}\text { Derecho a la } \\
\text { integridad de la } \\
\text { mujer violentada } \\
\text { psicológicamente } \\
\text { durante la } \\
\text { emergencia } \\
\text { sanitaria covid-19 }\end{array}$ & $\begin{array}{l}\text { Artículo } \\
\text { original }\end{array}$ & Ecuador & $\begin{array}{l}\text { Enfoque } \\
\text { cualitativo. } \\
\text { Métodos } \\
\text { inductivo, his- } \\
\text { tórico lógico } \\
\text { y analítico - } \\
\text { sintético }\end{array}$ & $\begin{array}{l}\text { Revisión } \\
\text { bibliográfica } \\
\text { jurídica }\end{array}$ & $\begin{array}{l}\text { Disminución de } \\
\text { la autoestima y } \\
\text { pobre percepción } \\
\text { de su valor } \\
\text { personal } \\
\text { incapacidad }\end{array}$ \\
\hline $\begin{array}{l}\text { vulnerables a la } \\
\text { violencia de género } \\
\text { en tiempos de } \\
\text { confinamiento }\end{array}$ & $\begin{array}{l}\text { Documento } \\
\text { de trabajo }\end{array}$ & España & $\begin{array}{l}\text { Análisis } \\
\text { estadístico de } \\
\text { indicadores }\end{array}$ & $\begin{array}{l}\text { Índices } \\
\text { sintéticos }\end{array}$ & $\begin{array}{l}\text { Aumento de } \\
\text { la presión } \\
\text { y el estrés. } \\
\text { Sentido de la } \\
\text { independencia } \\
\text { limitada }\end{array}$ \\
\hline $\begin{array}{l}\text { Violencia de } \\
\text { género en tiempos } \\
\text { de pandemia y } \\
\text { confinamiento }\end{array}$ & $\begin{array}{l}\text { Artículo de } \\
\text { revisión }\end{array}$ & España & $\begin{array}{l}\text { Investigación } \\
\text { documental }\end{array}$ & $\begin{array}{l}\text { Revisión } \\
\text { documental }\end{array}$ & $\begin{array}{l}\text { Depresión, } \\
\text { ansiedad }\end{array}$ \\
\hline
\end{tabular}

Fuente: elaboración propia. 
Balluerka (2020) planteó dos objetivos principales; uno, que busca "aportar información en profundidad sobre las consecuencias psicológicas del confinamiento por el estado de alarma" (p. 9), y otro, "centrado en analizar los efectos psicológicos de la pandemia y del confinamiento a nivel poblacional" (p. 9). La investigación se adelantó en dos fases: la primera, cualitativa, con entrevistas a profundidad; y una segunda fase cuantitativa, a partir de la aplicación de una encuesta para contrastar los efectos psicológicos producto del confinamiento dados en España y en otros países. Para la aplicación de la entrevista se realizó un muestreo intencional para obtener un número entre 30 y 40. La entrevista se realizó en nueve bloques que incluyeron actividad laboral, variables contextuales, percepción sobre la COVID-19 y el confinamiento, impacto del Lock Down o confinamiento en hábitos básicos, conciliación, impacto emocional, apoyo social y estrategias de afrontamiento, autocontrol y valoración final. La encuesta incluyó las siguientes secciones: actividad laboral, convivencia y apoyo, percepción de cambios personales, hábitos y actividades, situación en relación con la pandemia y vivienda.

La mayoría de las personas entrevistadas manifestaron cambios disfóricos, tales como resignación, tristeza, agobio, actitud crítica, decaimiento, entre otros. En relación con la encuesta, una de cada tres personas experimentó algo de dificultad para concentrarse. El $16 \%$ de mujeres y el 7,8 \% de hombres informaron un gran aumento en esta capacidad. El $36 \%$ de los hombres y el $48 \%$ de mujeres presentaron más sentimientos depresivos. La tendencia de "mucho incremento" de tales síntomas fue de predominio femenino, con un $7,6 \%$ de hombres y un 11,9\% de mujeres. Las participantes informaron con más frecuencia sentimientos de culpa (17\%) en comparación con los hombres $(10,4 \%)$. Un $4.1 \%$ de mujeres y un 1,3\% de hombres registraron "mucho incremento" en dicha culpabilidad (Balluerka et al., 2020).

El informe de Balluerka (2020) no analizó los efectos psicológicos de la violencia de género, lo que conduce a que únicamente haga recomendaciones en torno a dar y recibir apoyo social para superar las afectaciones evidenciadas.

Casique (2020) señala, que la confianza manifiesta en torno a las autoridades gubernamentales mexicanas por parte de las mujeres que conviven con parejas violentas había aumentado levemente en las dos últimas décadas. Sin embargo, el presidente mexicano, Manuel López Obrador, el 15 de mayo del 2020, expresó que el "90 \% de las llamadas al 911 por violencia contra las mujeres son falsas" (Casique, 2020). El análisis del impacto de tal declaración encontró que las mujeres, cuando buscan ayuda posterior a una agresión, lo 
hacen preferiblemente mediante canales informales, obviando la ayuda de carácter institucional. Más aún, el desconocimiento de la ruta de apoyo y ayuda institucional se considera un reflejo de la desconfianza infundida a las mujeres, de su frecuente revictimización, así como de la poca eficacia y eficiencia de los procedimientos respectivos.

También se evidenció el incremento en México de llamadas al 911 por eventos de violencia contra las mujeres en un $20.45 \%$, en el período comprendido entre febrero y marzo de 2020, con un pico máximo histórico para el mes de marzo de 2020. La autora concluye que, más allá de las cifras, es necesario fortalecer la confianza en las instituciones enfatizando la importancia de desnaturalizar la idea de que la violencia contra las mujeres es normal (Casique, 2020).

El estudio realizado por Chamba, Erazo, Narváez y Pinos (2020) analizó, desde el campo jurídico, el otorgamiento de las medidas de protección a las mujeres víctimas de violencia psicológica en el cantón Cuenca (Ecuador) durante el estado de emergencia por el coronavirus, en 2020. Para, ello asumió como como referente teórico el concepto de integridad personal como garantía constitucional, el cual:

se entiende como un conjunto de condiciones que permiten a las personas su existencia dentro de la sociedad, sin sufrir ningún tipo de violencia ni menoscabo de sus derechos en cualquier ámbito e índole, poniendo énfasis en las personas que se encuentra en un estado de vulnerabilidad y riesgo, por lo que la protección es una responsabilidad estatal. (Chamba et al., 2020, p. 92)

Con un muestreo por conveniencia, se aplicaron cuestionarios a tres funcionarios de las unidades judiciales de violencia intrafamiliar de Cuenca, dos defensores públicos, un juez; y diez abogados en libre ejercicio de la profesión. El 84.6 \% de dichos participantes consideró que las medidas protección no evitan, ni impiden que se presente la violencia psicológica en la emergencia sanitaria, producto de la dificultad para obtener pruebas, aunada a la ciberviolencia de género, para la cual existen muy pocos mecanismos de restricción. Como conclusión, el estudio plantea que, durante la emergencia por el COVID-19, se han evidenciado multiplicidad de casos de violencia psicológica, pero la legislación en Ecuador no responde de manera efectiva para proteger el derecho de integridad personal de las mujeres víctimas (Chamba et al., 2020). 
Gallo y Mañas (2020), en España, compararon los índices de vulnerabilidad territorial a la violencia de género a partir de índices de vulnerabilidad regional y provincial, teniendo en cuenta, igualmente, patrones espaciales y temporales. Para las autoras, la vulnerabilidad de las mujeres aumenta cuando se presentan situaciones de conflicto y desastres naturales. El contexto actual profundiza aún más las inequidades preexistentes, destacando “(...) el aislamiento social y la elevada carga que soportan las mujeres en las tareas de cuidados, ambos magnificados por el confinamiento (...)" (Gallo \& Mañas, 2020 , p. 9). El concepto de la "violencia de puertas para adentro" establece como potenciadores no solo las características individuales y las formas de relación, sino las condiciones habitacionales, en relación con las características del territorio habitado, como la pobreza, el desempleo, el bajo nivel educativo y de ingresos, así como el desorden físico del territorio.

El uso de estos índices sintéticos de vulnerabilidad territorial a la violencia de género permite cubrir varios aspectos a partir de información institucional; de igual forma, la multidimensionalidad del fenómeno consiente el uso de varios indicadores. Los resultados obtenidos para el año de 2015 indicaban que “(...) 1,7 millones de mujeres se están viendo obligadas a permanecer confinadas bajo el mismo techo, las 24 horas del día, con quienes hasta ahora habían venido ejerciendo violencia sobre ellas" (Gallo\& Mañas, 2020, p. 36), cifras que se incrementaron de manera sustancial en el contexto de la pandemia por COVID-19 de 2020.

El artículo de Lorente (2020) plantea cómo el confinamiento por causa del COVID-19 incrementó los factores de riesgo de violencia de género debido al aislamiento y a las barreras que dificultan la denuncia y el apoyo en dichas circunstancias. En una primera fase, durante el confinamiento, se incrementaron las diferentes formas de manifestación de la violencia (física, psicológica y sexual), y en una segunda fase, posterior al aislamiento, tras la pérdida de la sensación de control, se aumenta la posibilidad de una agresión letal a la mujer.

El encierro posibilita más el control abusivo del espacio de movilidad de la mujer, lo cual entorpece su posibilidad de huida. Tras eventos catastróficos, diversas investigaciones han dado cuenta de la presencia de violencia de género en relaciones en las que este fenómeno no existía. En cuanto a las consecuencias médico-legales del confinamiento desde lo psicológico, se debe considerar el tiempo que la mujer lleve sometida a las situaciones desestabilizadoras. En una primera fase domina la ansiedad, la cual posiblemente evolucione hacia la 
depresión (Lorente, 2020). Por ello, se recomienda incluir el riesgo de suicidio en las valoraciones médicas.

El deterioro de la salud de las mujeres que sufren violencia de género durante el confinamiento ocasiona el aumento de la demanda médica "(...) sin que sean las lesiones los elementos que caractericen el cuadro" (Lorente, 2020, p. 143). Entonces es necesario establecer una estrategia para detectar los casos de esta violencia, con el fin de adoptar las medidas para prevenir y mitigar el riesgo de nuevas agresiones, incluyendo las de carácter letal.

Los textos analizados dan cuenta de una similitud entre el confinamiento y los desastres naturales y los conflictos, con una mayor posibilidad de que se presente violencia de género de nuevo o que se incremente la ya existente. Esta semejanza con los desastres naturales le da mayor sustento a la declaración de crisis humanitaria, como se expone más adelante. Se resalta la posibilidad de un control mayor sobre la movilidad, libertad e identidad de la mujer por parte del agresor, lo que ocasiona estados de ansiedad y depresión, e incluso suicidios. Frente a la institucionalidad, se presenta una fuerte desconfianza debido principalmente a la normalización de los comportamientos, a la inadecuada preparación de los funcionarios o, en su defecto, al desconocimiento por parte de las víctimas de las rutas de atención.

\section{Acceso a la justicia con perspectiva de género en tiempos del COVID-19}

La crisis sanitaria, económica y social desatada por la pandemia en América Latina es sustancialmente diferente a las experiencias en países desarrollados, ya que los mayores niveles de inequidad preexistentes se han acentuado y las brechas sociales, económicas y de género se han ampliado, lo que supone una inestabilidad mayor en los próximos años. Al respecto, la Articulación Regional Feminista (2020) señaló que las decisiones de los gobiernos nacionales en la región no han tenido en cuenta la perspectiva de género, ni el impacto diferencial de la pandemia (p.13).

La Corte Constitucional colombiana, en diversas sentencias, ha hablado de la necesidad de incorporar la perspectiva de género en las decisiones judiciales. La Sentencia T-012 de 2016 recoge los diferentes criterios obligatorios que han de observarse entre los que se encuentran: i) investigación diligente y 
exhaustiva; ii) análisis hermenéutico de los hechos, pruebas y normas que reconozcan la discriminación histórica de las mujeres; iii) decisiones sin estereotipos de género; iv) evitar revictimización; v) flexibilizar la carga de la prueba; vi) considerar el rol transformador o perturbador de las decisiones judiciales; vii) análisis rígido sobre las actuaciones de la víctima viii) evaluar las posibilidades reales de los recursos y trámites judiciales, y ix) analizar las relaciones inequitativas que afectan la autonomía de las mujeres. Criterios que garantizan el acceso a la justicia con perspectiva de género. (p. 39).

En Colombia, el Ministerio de Salud y Protección Social declaró la emergencia sanitaria por COVID-19 el 12 de marzo de 2020. Acto seguido, el 22 del mismo mes, el Ministerio del Interior decretó el primer aislamiento preventivo obligatorio para los adultos mayores y el cese de actividades académicas presenciales en los colegios y universidades oficiales (Presidencia de la República de Colombia, 2020b). Posteriormente, el Gobierno decidió limitar el desplazamiento en el espacio público para el resto de los sectores de la población entre el 25 de marzo y el 13 de abril, con algunas excepciones derivadas del derecho a la vida y supervivencia de todas las personas.

Estas limitaciones se fueron flexibilizando, más por propiciar la reactivación económica que, por el curso epidemiológico de la pandemia en el país (Pinzón, 2020), que en agosto de 2020 alcanzó el primer pico (Johns Hopkins Coronavirus Resource Center, 2020). Así, a partir del 1 de septiembre de 2020 se declaró un aislamiento selectivo, en el cual la población general ya no está en confinamiento y solo hay restricciones a las aglomeraciones y a ciertas actividades (Presidencia de la República de Colombia, 2020d).

En esta misma línea, bajo la sombra del Artículo 215 de la Constitución Política de 1991, se declaró el Estado de Excepción el 17 de marzo de 2020, lo que implica una grave calamidad pública (Constitución Política de Colombia, 1991), derivada de la nueva enfermedad viral (Presidencia de la República de Colombia, 2020a). En esta declaratoria, el Gobierno consideró elementos de análisis relacionados con la salud pública y los aspectos económicos de orden nacional e internacional.

En el marco de la primera declaratoria de emergencia se tomaron medidas en todos los ámbitos del Gobierno. En relación con el acceso a la justicia, el 22 de marzo de 2020, el Gobierno nacional expidió el Decreto legislativo 460, "por el cual se dictan medidas el servicio a cargo de las comisarías de familia, 
dentro del Estado de Emergencia Económica, Social y Ecológica” que tiene en cuenta en sus consideraciones:

- Cifras publicadas por el Instituto Nacional de Medicina Legal y Ciencias Forenses (2020), que señalan a las mujeres como las principales víctimas del delito de violencia intrafamiliar y en específico de la violencia de pareja.

- Las recomendaciones de ONU Mujeres a los estados para generar las herramientas necesarias con miras a garantizar el acceso a la justicia de las mujeres, "facilitar la denuncia y solicitud de protección, en el marco de la incorporación del enfoque de género en la respuesta a la crisis generada por el coronavirus COVID-19” (Presidencia de la República de Colombia, 2020c).

- La Comisión Interamericana de Derechos Humanos recomendó a los estados parte: "Fortalecer los servicios de respuesta a la violencia de género, en particular la violencia intrafamiliar y la violencia sexual en el contexto de confinamiento" (Comisión Interamericana de Derechos Humanos, 2020, p. 17).

- Aumento de las llamadas a la línea de atención nacional 155 por denuncias por violencia intrafamiliar en el año de 2020, en comparación al año 2019(Observatorio de las mujeres, 2020a).

El Decreto 460 (2020c) expidió disposiciones acerca de la instauración de criterios de priorización del servicio y de atención presencial en casos excepcionales que abarcan "riesgo de feminicidio, violencia y acoso sexual, violencia psicológica y física, de amenazas o hechos de violencia" contra las mujeres, como se dispone expresamente en su Artículo 1. Mediante este decreto, el Gobierno nacional también pretende garantizar:

- La prestación del servicio ininterrumpido en las comisarías de familia.

- La continuación de audiencias de conciliación extrajudicial en derecho.

- El funcionamiento de policía judicial en las comisarías.

- La campaña de prevención de la violencia intrafamiliar.

- La obligatoriedad de las medidas.

De esta forma, el Decreto 460 (2020c) refuerza obligaciones que se encuentran incorporadas en el ordenamiento jurídico colombiano. Sin embargo, en la praxis se evidencia la imperiosa necesidad de medidas más contundentes, partiendo de un análisis contextual y diagnóstico de la situación de las mujeres en el país, orientado por la perspectiva de género. Estas normas idealmente deben incorporar presupuestos de acción política para los entes territoriales, como la definición de unas directrices claras para priorización urgente de un presupuesto nacional, y en los ámbitos departamental y municipal, para la intervención 
de las violencias contra las mujeres y el diseño e implementación de un plan de acción para todos los niveles administrativos del Estado, esto, dirigido al mismo objetivo. Estos serían dos ejes de actuación en los cuales, si se ofrece una formulación con una perspectiva de género real y efectiva, se centrarían los mismos postulados rectores que consisten en, primero, considerar que el acceso a la justicia de las mujeres es un derecho fundamental, y en consecuencia, su materialización y garantía no pueden suspenderse por un estado de emergencia. En segundo lugar, se debe asumir la obligación jurídica de que las violencias contra las mujeres son un asunto público que no se puede dejar en el ámbito de lo privado, para no darles el debido trámite e intervención.

Todas estas cuestiones son indispensables para garantizar el derecho de acceso a la justicia a las mujeres en todo momento, incluyendo los estados de emergencia. Adicionalmente, el Consejo Superior de la Judicatura emitió una circular dirigida a los juzgados de familia, para señalar que, por el estado de emergencia, no se admitían excusas para evadir el pago de cuotas alimentarias, "ya que estas constituyen un derecho fundamental de niñas, niños y adolescentes" (Consejo Superior de la Judicatura de Colombia, 2020) Así, eliminó algunos procedimientos para su autorización y habilitó su cobro de forma virtual.

Estas acciones en conjunto son importantes también para contribuir a disminuir las brechas de género que persisten y que se han acrecentado durante la crisis. Pero pueden fracasar si no se genera una red de acompañamiento institucional que reconozca los diferentes tipos de violencias contra las mujeres.

Como se ha expuesto, el sistema judicial en Colombia en su configuración y funcionamiento adolece de una perspectiva de género y de un enfoque de derechos humanos que permita intervenir de forma adecuada la prevención, atención, sanción, investigación y reparación de todas las formas de violencia contra las mujeres.

Lo anterior se materializa en situaciones como: reducida capacidad institucional para la administración de justicia, destinación presupuestal sin enfoque poblacional, prestación de servicios judiciales y de atención sin enfoque territorial, operadores jurídicos $\mathrm{y} / \mathrm{o}$ funcionarios/as públicos sin enfoque de género, política criminal sin perspectiva de género, entre otras.

Estas situaciones eventualmente dan lugar a presupuestos de hecho para establecer una violencia institucional por parte del Estado en contra de las mujeres 
víctimas de violencia. Estos presupuestos consisten, por ejemplo, en la dificultad y los obstáculos con los cuales se encuentran las mujeres para denunciar, la revictimización a las que se ven expuestas, la falta de protección efectiva, el alto costo de los procesos judiciales, los altos niveles de impunidad, la ausencia de respuestas administrativas y judiciales en relación con los procesos, la demora para impartir justicia, entre otras circunstancias específicas.

En este contexto de crisis sanitaria son las mujeres una de las poblaciones más afectadas, a las cuales se le reduce la protección de derechos fundamentales como lo es el derecho al acceso a la justicia. Las medidas restrictivas de emergencia para enfrentar la propagación del virus son una causa directa en el incremento de brechas y obstáculos para las mujeres acceder a la protección cautelar del Estado.

Esta pandemia también devela, de forma ineludible y contundente, lo que varias organizaciones feministas del país han nombrado y movilizado, frente a la masividad de las violencias perpetradas contra las mujeres. Estas son un problema público del cual debe ocuparse el Estado, que es severo y que constata que la casa no es un lugar seguro para las mujeres y las niñas. En este contexto, el acceso a la justicia se convierte en una garantía de vida o muerte para ellas.

Las medidas decretadas por el Gobierno nacional en el marco de la emergencia sanitaria afectaron la prestación del servicio de administración de justicia. Estas medidas han significado una reducción de la capacidad institucional que paraliza muchos procesos de violencias contra las mujeres. Asimismo, el confinamiento lleva a que las mujeres tengan menores posibilidades para denunciar. El acceso a la información es limitado en relación con las alternativas otorgadas para acceder a la justicia en el contexto actual, hay alta saturación de las líneas de atención, ausencia o restricción en la orientación y atención psicojurídica, falta de articulación institucional territorial para la garantía del acceso a los servicios de atención y recorte de los recursos para el fortalecimiento de los servicios públicos en este sentido.

Diferentes órganos institucionales nacionales han dispuesto algunos sistemas para recibir quejas y reclamos frente a la atención de violencias contra las mujeres, sobre todo en lo atinente a la violencia intrafamiliar, uno de estos órganos es la Procuraduría General de la Nación (2020), que señala:

durante el aislamiento obligatorio, se han recibido entre el 5 de abril de 2020 y el 22 de mayo de 2020, 551 quejas de la ciudadanía, de las cuales, 73 
corresponden a quejas por presuntas fallas en la atención de las violencias en razón del género y el sexo, en muchos de estos casos las mujeres víctimas advierten encontrarse en riesgo de feminicidio. (p. 4)

De esta forma, la falta de acceso a la justicia de una forma integral y garantista en circunstancias normales y, sobre todo, bajo un contexto de crisis, es un hecho que incrementa, de forma inminente, el riesgo de vulneración del derecho a la integridad personal y el derecho a la vida de las mujeres en todo el territorio nacional. En esta vía, el Estado, como primer garante del acceso a la justicia de las mujeres, ha de cumplir su obligación de debida diligencia, asunto del que no puede prescindir en estado de emergencia nacional; al contrario, se aumenta la exigibilidad para el cumplimiento de obligaciones jurídicas enmarcadas en la ley y en tratados internacionales de derechos humanos.

A partir de la orientación nacional del gobierno de turno, son las entidades departamentales y municipales quienes deben actuar y generar acciones más específicas que conciban, por ejemplo, que los servicios de atención a las violencias contra las mujeres sean servicios públicos de carácter esencial, que generan una mayor demanda a la capacidad institucional en medio de la crisis.

\section{De la crisis humanitaria a un estado de cosas inconstitucional: una declaración política que puede generar un reconocimiento jurídico}

El concepto de 'crisis humanitaria' ha sido reconocido en términos políticos tanto por los estados como por organismos internacionales para evidenciar la afectación o violación masiva de derechos humanos. Este término es traducido por la Corte Constitucional como el estado de cosas inconstitucional, para generar un reconocimiento jurídico y derivar consecuencias y responsabilidades a quienes permiten su configuración. Uno y otro acopian elementos similares: el primero, en foco político, y el segundo, en el constitucional.

El concepto 'crisis humanitaria' se ha utilizado usualmente para casos de desastres naturales, conflictos armados u aquellos eventos que implican una afectación masiva de derechos humanos. También está implícito un perjuicio global a las personas que tienen alguna condición relacional, han padecido dicha vulneración a raíz de una causa común y todo ello tiene un impacto político y social en el territorio donde se desarrolla. 
Teóricamente se ha analizado el concepto desde varias aristas. González (2015) hace un análisis desde la intervención de la ayuda humanitaria de Henry Dunant en los contextos de la violencia militar, desde la óptica de la ONU, bajo la premisa de que estas situaciones obedecen a colapsos totales o parciales de la autoridad estatal, que requieren intervención internacional, y, finalmente, cita a Susan Martin (2014), quien considera a la crisis humanitaria como

cualquier situación donde haya una amenaza generalizada a la vida, la seguridad física, salud o subsistencia básica que esté más allá de las capacidades de las comunidades en donde residen para resolverla. (...) Para entender por qué los efectos de un fenómeno natural o político se convierten en una crisis humanitaria es la debilidad institucional y, por lo tanto, la falta de gobernanza. (p.95)

Otros teóricos han planteado, que la crisis humanitaria puede tener origen en diversos factores económicos, sociales, demográficos, medioambientales, militares o políticos; y que dichas

crisis suelen darse en contextos de pobreza, fragilidad del estado y escasez alimentaria, en los que un desastre natural o un conflicto armado generan la aparición de una crisis alimentaria, enfermedades, desplazamientos forzados de población dentro del país o hacia el exterior, y una movilización importante de recursos internacionales en términos de ayuda. (Escola de Cultura de Pau, 2003, p. 43).

Si bien es cierto, que la génesis del término se circunscribe a contextos ligados con desastres naturales o conflictos armados, los elementos que caracterizan la crisis evidencian la relación entre afectación generalizada de derechos humanos en poblaciones históricamente discriminadas y la intervención estatal que adolece de gobernabilidad. Esto visibiliza la incapacidad estatal o la ineficacia del status quo en la intervención de la protección de la vida y la integridad física de las personas que habitan un territorio específico. Cuando se dan estas circunstancias, se exige una declaración política internacional o nacional, para que se inste a los gobiernos a tomar las medidas necesarias para salir de esa situación excepcional. Generalmente las realizan organizaciones internacionales, para advertir la precariedad de la situación y la urgencia de intervenir con medidas excepcionales. En el ámbito nacional, se procede de dicha manera cuando las afectaciones generalizadas (desastres naturales o pandemia) son evidentes o por la presión política de las personas afectadas (desplazamiento forzado). 
Estas declaraciones políticas se han llevado al debate jurídico, cuando los gobiernos no asumen por iniciativa propia esa responsabilidad. En Colombia, estas situaciones se han interpretado jurídicamente como un estado de cosas inconstitucional, por la Corte Constitucional, que, independiente del espacio constitucional ${ }^{1}$ en el que se reconozca, debe cumplir con los siguientes elementos: 1) vulneración masiva y generalizada de varios derechos constitucionales que afecta a un número significativo de personas; 2) prolongada omisión de las autoridades en el cumplimiento de sus obligaciones para garantizar los derechos; 3) adopción de prácticas inconstitucionales, como la incorporación de la acción de tutela como parte del procedimiento para garantizar el derecho conculcado; 4) no expedición de medidas legislativas, administrativas o presupuestales necesarias para evitar la vulneración de los derechos; 5) existencia de un problema social cuya solución compromete la intervención de varias entidades, requiere la adopción de un conjunto complejo y coordinado de acciones y exige un nivel de recursos que demanda un esfuerzo presupuestal adicional importante; y 6) si todas las personas afectadas por el mismo problema acudieran a la acción de tutela para obtener la protección de sus derechos, se produciría una mayor congestión judicial (Corte Constitucional de Colombia, 2004).

Rodríguez Garavito (2010) propone resumir estos elementos en: 1) las condiciones del proceso: fallas estructurales de las políticas públicas en el país; 2) condiciones de resultado: violación masiva y sistematizada de los derechos fundamentales de un número indeterminado de personas; 3) la necesidad imperiosa del trabajo en conjunto de diversas autoridades para la modificación. El análisis se hace a partir de estos tres componentes que recogen tanto la crisis humanitaria como el Estado de Cosas Inconstitucional. A continuación, la descripción de los elementos enunciados.

\section{Fallas estructurales de las políticas públicas en el país}

Aunque se han hecho algunos esfuerzos exigidos desde los movimientos de mujeres y la bancada de mujeres en el congreso, existen fallas estructurales por la inexistencia de una política criminal con perspectiva de género, con presupuesto específico para intervenir la problemática; aunque hay un trazador presupuestal "vida libre de violencias", no necesariamente hay indicadores de resultado que cuantifiquen la erradicación de las violencias contra las mujeres

Sistema penitenciario, poblaciones indígenas, régimen pensional, defensores y defensoras de derechos humanos, desplazamiento interno forzado. 
en el país. Además, faltan órganos especializados en todo el país (tanto en lo urbano como en lo rural) que investiguen, de manera diligente, estas violencias. Finalmente, a pesar del acervo normativo de género, su aplicación es baja.

\section{Violación masiva y sistematizada de los derechos fundamentales de un número indeterminado de personas}

La violencia contra las mujeres constituye una violación sistemática y permanente en Colombia. En condiciones "normales", es decir, fuera de la pandemia por COVID-19, las violencias contra las mujeres configuran un problema político y de salud pública (Ramírez \& Ariza, 2015) e incluso una preocupante endemia si se analiza en términos epidemiológicos. En la pandemia, esta violencia se exacerbó: las llamadas al 155 se aumentaron en un $99 \%$ en el periodo del 25 de marzo al 29 de octubre de 2020, con respecto al año 2019 (23811 llamadas en el 2020, 94 \% en cabeza de mujeres). Las llamadas por violencia intrafamiliar en este mismo lapso se incrementaron en $116 \%$, con un registro de 18034 en el 2020 (Observatorio de las Mujeres, 2020b). Con respecto a los feminicidios, entre marzo y septiembre de 2020 se han presentado 357 casos en Colombia. Se informaron 16 transfeminicidios en ese periodo, al rectificar el subregistro, pues hubo casos que se anotaron inicialmente como hombres (Observatorio de feminicidios Colombia, 2020b).

En cuanto a la violencia sexual, de enero a abril de 2020 se realizaron 6346 exámenes médicolegales por presuntos casos. Del total, 5398 correspondieron a mujeres, es decir, el 85,06 \%, y 948 a hombres, esto es, el 14,94 \% (Sisma Mujer, 2020).

\section{La necesidad imperiosa del trabajo en conjunto de diversas autoridades para la modificación}

Aunque en el contexto de la pandemia se expidieron algunas disposiciones para intervenir las diversas violencias, el 26 de junio de 2020 se adelantó un consejo de seguridad nacional para analizar la situación, y se tomaron ocho medidas para intervenir la problemática². Se evidencia que no hay un

\footnotetext{
i) Instalación de una sala de control y monitoreo exclusiva para combatir crímenes de violencias en contra de las mujeres. ii) Consejo de seguridad nacional. iii) Plataforma tecnológica con inteligencia artificial para prevención, atención y seguimiento de estas violencias. iv) Unificación de las líneas telefónicas encargadas de atender el tema. V) Expedición de un decreto para formalizar
} 
presupuesto destinado de manera clara, ni una articulación estructural a lo largo del territorio colombiano. De igual forma, los municipios de menor categoría y la ruralidad son los menos intervenidos y se observan inequidades que amplían las brechas sociales y de género ya existentes (Vicepresidencia de la República de Colombia, 2020).

\section{Conclusiones}

Ahora que se habla de sindemia para referirse al empeoramiento de las epidemias cuando se potencian sus efectos negativos por condiciones como la pobreza, la discriminación y las exclusiones (Lolas, 2020), es posible interpretar la violencia contra las mujeres en 2020 como un ejemplo de este fenómeno. Esto, porque los efectos negativos de esta violencia se agravan por la interseccionalidad de la categoría género (Esguerra y Bello, 2014), con otras, como orientación sexual, condiciones socioeconómicas, etnia, procedencia, migración y desplazamiento.

Las cifras y datos en el contexto internacional, también evidentes en el caso colombiano, dan cuenta del incremento de la violencia de género contra las mujeres por parte de sus parejas durante el confinamiento producto del COVID-19, quizás debido a la facilidad para que el agresor logre tener un mayor control sobre su pareja, en cuanto a su movilidad y libertad de acción. A pesar de la legislación existente y las medidas adoptadas por parte del Gobierno colombiano, las cifras de llamadas a líneas de emergencia se elevaron, pero no así las denuncias, producto también de la desconfianza en la capacidad estatal para la atención y protección de las mujeres.

La dimensión psicológica de la violencia de género ha sido estudiada de manera tal que ha quedado oculta por otros síntomas y fenómenos que afectan de igual forma a las mujeres durante este período. Aunque entre los efectos que se han podido evidenciar están la ansiedad y la depresión agudas, esta última puede ser determinante para la presencia de un cuadro depresivo estructural. Es recomendable, para los profesionales que atienden estos casos, analizar la

\footnotetext{
el mecanismo e implementación de una campaña nacional. Vi) Radicación de un proyecto de ley para el fortalecimiento de las comisarías de familia. vii) Implementación de protocolos para la prevención y atención del acoso sexual en las universidades. Y viii) programa para la estabilización socioeconómica de las mujeres víctimas de violencias de manera conjunta con el Ministerio de Trabajo y el Ministerio de Comercio, Industria y Turismo (Vicepresidencia de la República de Colombia, 2020).
} 
posibilidad de ocurrencia del suicidio. Las afectaciones psicológicas producidas por el confinamiento son similares a las descritas en contextos de conflicto y desastres naturales. A lo descrito, se le suma la incapacidad institucional y legislativa, así como la dificultad de comprobar la afectación en la dimensión psicológica. Para el caso colombiano, se hace indispensable elaborar estudios e investigaciones que profundicen en el fenómeno y que brinden insumos para los entes ejecutivo y legislativo, para la toma de decisiones y la realización de acciones que enfrenten esta otra pandemia - o sindemia- que se incrementa de manera progresiva.

El acceso a la justicia para las mujeres en el país es una de las esferas cuyo análisis arroja unas manifestaciones expresas de las brechas existentes entre hombres y mujeres desde lo social, político, económico y cultural, las cuales tienen bases que se consolidan dentro de un sistema regido por relaciones desiguales de poder que generan estereotipos, discriminaciones y violencias.

El Estado colombiano ha ratificado, a partir de 1996, un número importante de instrumentos internacionales en materia de reconocimiento, protección y garantías de los derechos de las mujeres. Todo esto se ha materializado en diferentes leyes y políticas públicas. Si bien es cierto que la pandemia por COVID-19 ha puesto en jaque la materialización de los ideales plasmados en los textos, es menester que el Gobierno nacional revise cómo la promulgación de decretos y resoluciones puede impactar a largo plazo la vida de mujeres y niñas, sin tener que esperar a que la Corte Constitucional tome medidas de facto que le corresponden a las políticas públicas que expide el gobierno.

Las violencias contra las mujeres en Colombia se constituyen en una crisis humanitaria por la afectación psicológica que producen en las víctimas, por su inadecuada atención y por el creciente número de casos. Se ha dado una respuesta estatal insuficiente para su erradicación, lo que requiere tanto una declaración política, como una declaración constitucional bajo el estado de cosas inconstitucional, para que se dicten las medidas administrativas, financieras y judiciales necesarias, para erradicar la crisis y así poder garantizarles realmente a las mujeres y niñas vidas libres de violencias. 


\section{Referencias}

Ariza, G. (2013). Las representaciones sociales de la violencia en las relaciones de pareja en Medellín en el siglo XXI. Revista CES Psicología, 6(1), 134-158. Recuperado de: http://revistas.ces.edu.co/index.php/psicologia/article/view/2577

Articulación Regional Feminista, ARF. (2020). Los derechos de las mujeres de la región en épocas de COVID-19. Estado de situación y recomendaciones para promover políticas con justicia de género. Buenos Aires. (archivo PDF) Recuperado de: http:// www.articulacionfeminista.org/a2/objetos/adjunto.cfm?aplicacion=APP003\&cnl= 26\&opc $=7 \&$ codcontenido $=4411 \&$ codcampo $=25$

Ballester, A., \& Villanueva, L. (2014). Valoración psicológica en delitos de violencia de género mediante el Inventario Clínico y Multiaxial de Millon III (MCMI-III). Anuario de Psicología Jurídica, 24(1), 9-18. Recuperado de: https://doi.org/10.1016/j. apj.2014.06.001

Balluerka, N., Gómez, J., Hidalgo, M. D., Gorostiaga, A., Espada, P., Padilla, J. L., \& Santed, M. Á. (2020). Las consecuencias psicológicas de la COVID- 19 y el confinamiento. Informe de investigación. (archivo PDF). Recuperado de: https://www.ub.edu/web/ $\mathrm{ub} / \mathrm{ca} / \mathrm{menu}$ _eines/noticies/docs/Consecuencias_psicologicas_COVID-19.pdf

Carrasco, L., \& Martínez, M. J. (2020). Riesgos inminentes, cuerpos descorporizados, silencios que gritan, luchas colectivas o muerte: efectos de la pandemia COVID-19 en la configuración de las violencias contra las mujeres. Red Sociales, Revista del Departamento de Ciencias Sociales, 7(2), 46-57. Recuperado de: http://ri.unlu.edu. ar/xmlui/handle/rediunlu/749

Casique, I. (2020). Violencia. Notas de coyuntura del CRIM, 28. Recuperado de: https://web.crim.unam.mx/sites/default/files/2020-06/crim_028_irene-casique_ recrudecimiento-de-la-violencia.pdf

Castro, A. (2020). Desafíos de la pandemia de COVID-19 en la salud de la mujer, de la niñez y de la adolescencia en América Latina y el Caribe. PNUD América Latina y el Caribe, Unicef, COVID19. Serie de documentos de política pública. (archivo. pdf). Recuperado de: https://www.unicef.org/lac/media/16356/file/cd19-pdsnumber19-salud-unicef-es-003.pdf

Chamba, N., Erazo, J., Narváez, C., \& Pinos, C. (2020). Derecho a la integridad de la mujer violentada psicológicamente durante la emergencia sanitaria COVID-19. Iustitia socialis, 5(9), 89-111. Recuperado de: https://doi.org/10.35381/racji.v5i9.727

Comisión Interamericana de Derechos Humanos. (2020). Pandemia y derechos humanos en las Américas. Resolución 1/2020. Adoptada por la CIDH el 10 de abril de 2020. OEA. Recuperado de: https://www.oas.org/es/cidh/decisiones/pdf/Resolucion-120-es.pdf

Congreso de la República de Colombia. (1994, 31 de mayo). Ley 137. Por la cual se dictan normas sobre Mecanismos de Participación Ciudadana. Diario Oficial 41373. https://www.funcionpublica.gov.co/eva/gestornormativo/norma.php?i=330

Consejo Superior de la Judicatura de Colombia. (2020). Medidas COVID-19: Autorizaciones de pago de títulos por concepto de alimentos (Circular PCSJC20-10 del 25 de marzo de 2020). Recuperado de: https://www.ramajudicial.gov.co/ documents/10228/3385454/CIRCULAR+ALIMENTOS.pdf/7c094119-b796-4458$810 f-40 \mathrm{~d} 6 \mathrm{ba0}$ ced 05 
Corte Constitucional de Colombia. (2004, 22 de enero). Sentencia T-025/04 (T-653010 y acumulados, magistrado Manuel José Cepeda Espinosa). Recuperado de: http:// www.corteconstitucional.gov.co/relatoria/2004/t-025-04.htm

Corte Constitucional de Colombia. (2016, 22 de enero). Sentencia T-012/16 (Magistrado Luis Ernesto Vargas Silva). Recuperado de: https://www.corteconstitucional.gov.co/ relatoria/2016/t-012-16.htm

Escola de Cultura de Pau. (2003). ¡Alerta 2003! Informe sobre conflictos, derechos humanos y construcción de paz. Universidad Autónoma de Barcelona: Icaria.

Esguerra, C., \& Bello, J. (2014). Interseccionalidad y políticas públicas LGBTI en Colombia: usos y desplazamientos de una noción crítica. Revista de Estudios Sociales, (49), 19-32. https://doi.org/10.7440/res49.2014.02

Gallo, M., \& Mañas, E. (2020). Territorios vulnerables a la violencia de género en tiempos de confinamiento. Documentos de Trabajo (IAES, Instituto Universitario de Análisis Económico y Social), (5), 1-42. Recuperado de: http://hdl.handle.net/10017/43144

González, E. (2015). Crisis humanitaria, violencia criminal y desplazamiento forzado en el Triángulo Norte de Centroamérica. Revista de Relaciones Internacionales de la UNAM, (122/123). Recuperado de: http://www.revistas.unam.mx/index.php/rri/ article/view/57439

Johns Hopkins Coronavirus Resource Center. (2020). Colombia -COVID-19 OverviewJohns Hopkins. Recuperado en noviembre 17, 2020, de: https://coronavirus.jhu. edu/region/colombia

Hale, T., Angrist, N., Cameron-Blake, E., Hallas, L., Kira, B., Majumdar, S., Petherick, A., Phillips, T., Tatlow, H., \& Webster Samuel. (2020). Stay-at-home requirements during the COVID-19 pandemic. Oxford COVID-19 Government Response Tracker, Blavatnik School of Government. Our World in Data. Recuperado de: https://ourworldindata. org/grapher/stay-at-home-covid

Instituto Nacional de Medicina Legaly Ciencias Forenses.(2020). Boletínestadístico mensual. Recuperado de: https://www.medicinalegal.gov.co/documents/20143/494197/ Boletin+enero.pdf/7498aebf-058a-0b33-f072-95e2a5b12c4b

Llerena, R., \& Narváez, C. (2020). Emergencia, gestión, vulnerabilidad y respuestas frente al impacto de la pandemia COVID-19 en el Perú. Recuperado de: https://doi. org/10.1590/SciELOPreprints.94

Lolas, F. (2020). Perspectivas bioéticas en un mundo en sindemia. Acta Bioethica, 26(1), 7-8. https://doi.org/10.4067/S1726-569X2020000100007

Lorente, M. (2020). Violencia de género en tiempos de pandemia y confinamiento. Revista Española de Medicina Legal, 46(3), 139-145. Recuperado de: https://doi. org/10.1016/j.reml.2020.05.005

Lozano, J. (2005). De patos, gansos y cisnes. Revisiones narrativas, revisiones sistemáticas y meta-análisis de la literatura. Acta Medica colombiana, 30(1), 1-4. Recuperado de: http://www.scielo.org.co/scielo.php?script=sci_abstract\&pid=S0120$24482005000100001 \&$ lng =en\&nrm=iso\&tlng=es

Observatorio de Feminicidios Colombia. (2020a). Boletín mensual junio. Valle de Aburrá. Ni una menos. Red feminista antimilitarista. Recuperado de: http:// www.observatoriofeminicidioscolombia.org/index.php/seguimiento/boletinregional/433-ni-una-menos-boletin-sobre-feminicidios-en-medellin-y-el-vallede-aburra-junio-de-2020 
Observatorio de Feminicidios Colombia. (2020b). Boletín mensual septiembre. Valle de Aburrá. Ni una menos. Red feminista antimilitarista. Recuperado de: https:// observatoriofeminicidioscolombia.org/attachments/article/442/Bolet\%C3\%A Dn\%20Vivas\%20Nos\%20Queremos\%20Septiembre\%202020.pdf

Observatorio de las Mujeres. (2020a). Llamadas para la orientación de mujeres en condición de vulnerabilidad. Boletín, 11. Recuperado de: http://www. observatoriomujeres.gov.co/archivos/publicaciones/Publicacion_20.pdf

Observatorio de las Mujeres. (2020b). Llamadas para la orientación de mujeres en condición de vulnerabilidad. Boletín, 31. Recuperado de: http://www. observatoriomujeres.gov.co/archivos/publicaciones/Publicacion_73.pdf

Perela, M. (2010). Violencia de género: violencia psicológica. Foro: Revista de Ciencias Jurídicas y Sociales, Nueva Época, 0(11), 353-376. Recuperado de: https://doi. org/10.5209/FORO.37248

Pinzón, J. (2020). Estudio comparativo entre el contagio durante la cuarentena obligada por el COVID-19 y el contagio durante la apertura gradual y controlada para algunos sectores de la economía en Colombia. Revista Repertorio de Medicina y Cirugía, edición especial, 52-58. Recuperado de: https://doi.org/10.31260/ RepertMedCir.01217372.1073

Presidencia de la República de Colombia. (2020a, 17 de marzo). Decreto 417. Por el cual se declara un estado de emergencia económica, social y ecológica en todo el territorio nacional. Diario Oficial 51259. https://www.funcionpublica.gov.co/eva/ gestornormativo/norma.php?i=110334

Presidencia de la República de Colombia. (2020b, 22 de marzo). Decreto 457. Por el cual se imparten instrucciones en virtud de la emergencia sanitaria generada por la pandemia del Coronavirus COVID-19 y el mantenimiento del orden público. Diario Oficial 51264. https://www.funcionpublica.gov.co/eva/gestornormativo/norma. php?i=110674

Presidencia de la República de Colombia. (2020c, 22 de marzo). Decreto Legislativo 460. Por el cual se dictan medidas el servicio a cargo de las comisarías de familia, dentro del Estado de Emergencia Económica, Social y Ecológica. Diario Oficial 51264. https://www.funcionpublica.gov.co/eva/gestornormativo/norma. php?i=110655Presidencia de la República de Colombia. (2020d, 4 de septiembre). Decreto 1168. Por el cual se imparten instrucciones en virtud de la emergencia sanitaria generada por la pandemia del Coronavirus COVID - 19, y el mantenimiento del orden público y se decreta el aislamiento selectivo con distanciamiento individual responsable. Diario Oficial 51427. https://www.funcionpublica.gov.co/eva/ gestornormativo/norma.php?i=140210

Procuraduría General de la Nación (2020). Directiva 022. Recuperado de: https://www. procuraduria.gov.co/relatoria/media/file/DIRECTIVA\%2022\%20DE\%202020\%20 (1).pdf

Ramírez, M., \& Ariza, G. (2015). Lo político de la violencia en las relaciones de pareja como problema de salud pública. Revista de la Facultad de Medicina, 63(3), 517525. Recuperado de: https://doi.org/10.15446/revfacmed.v63n3.45191

Rodríguez, C. (Ed.). (2010). Más allá del desplazamiento: políticas, derechos y superación del desplazamiento forzado en Colombia. Bogotá: Universidad de los Andes.

Scott, J. (2008). Algunas reflexiones adicionales sobre género y política. In C. Boadas (Trans.), Género e Historia (pp. 245-269). Fondo de Cultura Económica: Universidad Autónoma de la Ciudad de México. 
Sisma Mujer. (2020). En tiempos de pandemia tampoco es hora de callar la violencia contra las mujeres. Boletín (21). Recuperado de: https://www.sismamujer.org/ wp-content/uploads/2020/09/25-05-2020-Boleti\%CC\%81n-Sisma-Mujer-25-demayo-de-2020-.pdf

Trujillo, M. (2019). Misoginia y violencia hacia las mujeres: dimensiones simbólicas del género y del patriarcado. Atenea (Concepción), 519, 49-64. Recuperado de: https:// doi.org/10.4067/S0718-04622019000100049

Unicef. (2020). Encuesta de percepción y actitudes de la población. Impacto de la pandemia COVID-19 y las medidas adoptadas por el gobierno sobre la vida cotidiana. Buenos Aires: Unicef. https://www.unicef.org/argentina/media/8056/ file/Covid19-EncuestaRapida-InformeEducacion.pdf

UN (2020), Secretary-General's address to the Opening of the General Debate of the 75th Session of the General Assembly. Versión en español. https://www.un.org/sg/en/ content/sg/statement/2020-09-22/secretary-generals-address-the-opening-ofthe-general-debate-of-the-75th-session-of-the-general-assembly

Vicepresidencia de la República de Colombia (2020). Gobierno arrecia medidas para frenar violencia contra las mujeres. Recuperado de: https://mlr.vicepresidencia.gov. co/Paginas/prensa/2020/Gobierno-arrecia-medidas-para-frenar-violencia-contralas-mujeres.aspx 